\title{
Economía del conocimiento, conocimiento indígena y migración
}

\author{
LIEPOLLO LEBOHANG PHEKO*
}

RESUMEN: Para sostenerse, los turbulentos fenómenos encarnados actualmente por la globalización dependen del conocimiento y la innovación. Las fuerzas del mercado y el imperativo de la búsqueda de ganancias ponen en riesgo el plano de la innovación. El contexto de globalización y migración deriva en un mayor análisis del movimiento de la gente y en el impacto que ello pudiera tener en la pérdida de capacidades en el Sur global. El capitalismo del conocimiento es una de las fuerzas más poderosas del capital global y no tiene ningún interés en la distribución igualitaria de los beneficios sociales más allá de la ganancia o de la apreciación del conocimiento indígena. A lo largo de este texto el uso intencional de afrikano, en lugar de africano, es para expresar el posicionamiento filosófico del autor y de la escuela de pensamiento a la cual pertenece.

PALABRAS CLAVE: globalización, conocimiento afrikano indígena, migración, era de la información, modernidad.

\footnotetext{
* Directora Ejecutiva de The Trade Collective, Sudáfrica.

Traducción del inglés de Jorge Miguel Veizaga Rosales.
} 
ABSTRACT: The turbulent phenomena that currently is expressed as globalisation depends on knowledge and innovation for its sustenance. Market forces and the profit imperative leave little room for innovation. The context of globalisation and migration requires further scrutiny on the movement of people and the impact that this may have on skills drain in the global south. Knowledge capitalism is one of the most potent forces of global capital, which has no interest in the egalitarian distribution of social benefits beyond profit nor in the recognition of indigenous knowledge. Note the use of «Afrikan» rather than «African» throughout this paper, which is intended to convey the philosophical stance of the writer and the intellectual school of thought to which she belongs.

KEY WORDS: globalisation, Afrikan indigenous knowledge, migration, information age, modernity. 


\section{INTRODUCCIÓN}

T os términos "globalización» y «economía del conocimiento» están sujetos a un riguroso debate pero también el uso se presta a una gran confusión. En diversos contextos el constructo de globalización evoca múltiples significados. Más allá del significado restringido de globalización que describe, principalmente, la integración económica y financiera, este artículo sugiere y asume una definición más amplia que incluye varias esferas de la vida. Dicha óptica permite evaluar, además de los sistemas financieros, la dimensión social, cultural y geopolítica. Boler (2008) las describe como «esferas de la globalización». A pesar de que los Sistemas de Conocimiento Indígena Africano (SCIA) han existido por miles de años, su concepto y práctica comenzaron a aparecer en el ámbito de la ciencia hace sólo tres décadas. El desarrollo de nuevas realidades políticas, económicas y culturales, además de las metodologías posmodernas, crearon las bases y nuevas maneras de comprender y asumir el scia. Algunas de las nuevas realidades y desarrollos son el reconocimiento político de los pueblos indígenas, el fracaso de la planificación del desarrollo para alcanzar resultados deseados, la creciente desilusión de los afrikanos respecto a las promesas de la ciencia «occidental» moderna y, al mismo tiempo, el creciente interés público por el valor del patrimonio cultural y la idea de que la «ciencia» debe ubicarse en el contexto social y cultural (Nel, 2005).

Es muy importante evaluar, identificar y examinar los orígenes y desafíos clave que enfrenta el Sistema de Conocimiento Indígena (SCI) y explorar el déficit de credibilidad entre el ScI y la economía de mercado. El déficit es particularmente problemático dada la enorme dependencia y beneficios que el capitalismo global ha acumulado a partir del reservorio del sci.

En el contexto afrikano, el conocimiento o la economía de la información ofrece una oportunidad para desarrollar de manera autodefinida y relevante el uso y aplicación de los medios, culturas, conocimiento indígena, propiedad intelectual y tecnología, entre muchos puntos de entrada multidisciplinarios. Debemos tener muy en cuenta que, como en cualquier otro ámbito, la economía del conocimiento no tiene un valor neutro y a menudo es hostil a 
los intentos de los afrikanos para generar información. El género, como un constructo social, sería un punto para abordar una investigación más amplia acerca de la creación del conocimiento y la democratización de la información.

A pesar de que la creación de ideas es el punto focal, muchos países afrikanos y muchas comunidades del conocimiento no reciben el reconocimiento que corresponde a su contribución. En parte esto puede atribuirse a la transmisión, en gran medida no codificada, del conocimiento indígena y, también, al machismo patriarcal que persiste en muchas instituciones académicas y de investigación. El estudio y creación de conocimiento «duro» y tangible todavía se percibe como competencia del análisis occidental. El conocimiento ancestral y la sabiduría indígena, incluyendo el conocimiento afrikano indígena, han sido las bases de muchas actividades benéficas actuales donde las mujeres han sido no sólo las custodias de tal conocimiento, sino también repositorios y árbitros de su uso. Ahora han sido separadas de la continua investigación del conocimiento y excluidas de sus beneficios. En realidad, existe una pequeña dicotomía entre conocimiento y economías de mercado.

Para complicar aún más el terreno, los catalizadores de la economía del conocimiento están vinculados en gran medida a la ventaja competitiva de crear nuevas tecnologías y técnicas cada vez más eficientes para descubrir y aplicar información. La globalización en su forma más cruda ha usado la información y la distorsión para marginar y explotar naciones y pueblos que no tienen acceso irrestricto al conocimiento e iniciativa epistemológica codificada. El modelo usado durante la Revolución Industrial continúa usando tecnología para procurar la disponibilidad global y masiva de productos y servicios. La investigación, archivo y conservación de repositorios nacionales-regionales permite la investigación y desarrollo de una gama de soluciones para los desafíos locales en todas las esferas de las necesidades humanas. Los sacerdotes de la globalización constantemente intentan disminuir la primacía del Estado y las identidades nacionales y culturales, en particular de los africanos, argumentando que ello es anticompetitivo y presentando el discutible supuesto de que la competencia imperfecta puede generar consecuencias socioeconómicas perfectas y equilibradas. Conocimiento 
e identidad están estrechamente vinculadas y son esenciales para continuar la introspección y la exploración.

\section{IMPLICACIONES DEL CONOCIMIENTO INDÍGENA AFRIKANO Y LOS INDÍGENAS}

Es importante comprender el campo y la naturaleza del conocimiento indígena africano (CIA) además de su importancia, complejidad y contexto. Las definiciones normativas occidentales utilizan lo «indígena» como comparable o equivalente a «tradicional», «aborigen», «vernáculo», «africano», «negro»y «nativo-americano» (Loubser, 2003: 76).

La definición de indígena de Loubser alude a un grupo de personas que comparten normas comunes y cultura, que han ocupado un espacio geográfico particular por varias generaciones (Loubser, 2003: 75). Los grupos se involucran en el conocimiento y la práctica de sistemas de conocimiento que definen sus creencias, espiritualidad, patrimonio y - algo muy importante- los distinguen de otros grupos (Dondolo, 2005: 112; Odora-Hoppers, 2005; Nel, 2005; Masoga, 2005). Básicamente, los sCiA son «instruidos por e identificados con todas las áreas de la vida y la naturaleza» (Nel, 2005). Los discursos contemporáneos en relación con lo indígena, la pertenencia y la identidad afirman que: "Es ser lo que se espera, individuos que tienen numerosas personalidades para cubrirse moldeadas por el actual flujo monetario y político y su apariencia en el escenario social» (Nel, 2005).

Los elementos subyacentes al scia trabajan en dos dimensiones interconectadas: experimental y cognitiva. El nivel experimental puede ser dividido en los ámbitos: i) natural; ii) tecnológico y arquitectónico, y iii) sociocultural. La esfera natural comprende la biodiversidad, agricultura, ecología, suelo, medicina y farmacia. En segundo lugar, el campo de lo tecnológico y arquitectónico comprende todas las técnicas, tales como la metalurgia, los textiles, cestería, procesamiento de alimentos y construcción. Las dimensiones socioculturales de la vida incluyen gobernanza, resolución de 
conflictos, arte, música, bienestar social, todo ello hace a la tercera esfera (Odora-Hoppers, 2005: 3).

La mayor parte de estas acciones y ceremonias tienen lugar dentro de un contexto cultural que implica prácticas (algunas de las cuales incluyen canciones, bailes y moda) (Odora-Hoppers, 2005) y en armonía con la naturaleza. Esto contrasta con el concepto superficial de la realidad, el cual promueve una relación combativa e imperialista con la naturaleza (Capra, 1989: 226). Armonía, interdependencia e interrelación es el ejemplo del SCIA, ya sea social, cultural, espiritual, biológica o física. A pesar de que los SCIA están cultural y filosóficamente conectados, virtualmente todas las comunidades indígenas tienen una comprensión ontológica común que respeta todas las formas de vida y no separa ni privilegia a los humanos en la ecología de la existencia.

El concepto de los SCIA también define un abordaje racional a través del cual los modelos y precisiones tanto de la naturaleza como de la cultura son teorizados (Odora-Hoppers, 2005: 3). En consecuencia, la correlación entre el conocimiento indígena, sus poseedores y las tecnologías e instrumentos usados para su aplicación están unidos a una cosmología, una forma de ver el mundo (Nel, 2005). El núcleo de la cosmología indígena tiene que ver con «la co-evolución de los mundos espiritual, natural y humano» (OdoraHoppers, 2005: 4).

\section{¿QUÉ ES Y DÓNDE ESTÁ LA ECONOMÍA DEL CONOCIMIENTO?}

La adquisición y uso de la información han sido catalizadores esenciales del crecimiento y han contribuido crecientemente al producto interno bruto (PIB) durante los últimos sesenta años. La fluidez entre la aplicación y difusión del conocimiento es un pilar crucial para el crecimiento económico y se ha convertido en un pilar más importante aún durante la era del crecimiento acelerado en la economía del conocimiento. El crecimiento ocurre a través de la producción de información nueva por medio de su transmisión y utilización. La monetización de la información es más simple debido a los mode-

$96 \frac{\text { SEGUNDO SEMESTRE } 2015}{\text { MIGRACIÓN Y DESARROLLO NÚM. } 25}$ 
los de cadenas globales de valor que permiten reempacar la información existente para nuevos mercados. El valor económico de la información es comparable con el de la producción y la gestión. En esencia, hay varios tipos de información, cuyo alcance está más allá de la economía de mercado. Estos tipos incluyen: i) conocimiento práctico, e.g., negocios, cuestiones legislativas, funciones de los hogares; ii) aprendizaje aplicado, esto es, el estudio de eventos sociales y mediáticos y la satisfacción de la curiosidad académica; iii) ocio, es decir, información que satisface el interés no académico o el ocio; $i v)$ interés filosófico o religioso, y v) aprendizaje casual, obtenido sin darse cuenta (Machlup, 1962: 13, 21-22).

Los economistas y especialistas de la administración identificaron inicialmente un componente clave de información para el desarrollo financiero y social a finales de los años cincuenta y mediados de los sesenta. Como resultado, esto se expandió al campo de la sociología con el intento de problematizar las formas cambiantes de interacción social. La expresión «economía de la información» fue creada por Fritz Machlup (1962) en el trabajo «La producción y distribución de conocimiento en los Estados Unidos» donde cuestiona que la información constituya una innovación en Estados Unidos (Cortada, 1998). Él define la información como «cualquier acción humana (o instigada por el hombre) adecuadamente destinada a crear, modificar o afirmar en una personalidad humana la propia personalidad de uno o la de cualquier otro individuo, una conciencia, discernimiento o atención significativa» (Machlup, 1962: 30).

La aplicación de la economía del conocimiento no está confinada a la interdependencia electrónica, a un mayor alcance de mercado y uniformidad de la producción ni a los gustos del consumidor. Más bien se relaciona fuertemente con el autoconocimiento, autoconciencia y autopreservación de Áfrika. Las franquicias de productos que vemos hoy en los supermercados, centros comerciales, pantallas de televisión, estaciones de radio y revistas ilustran el éxito generalizado de la «marca global». Siendo las mujeres las principales custodias de este conocimiento y teniendo el desafío de acceder y beneficiarse de la información, resulta importante colocar en el centro del análisis sus preocupaciones y perspectivas. 
La economía del conocimiento normalmente prefiere usar el capital intelectual como mecanismo de expansión, mientras que la economía de mercado prefiere el capital financiero, que puede, por ejemplo, ser comprado, vendido, movido, cambiado, agregado en uniones o expansiones. Además, la economía del conocimiento tiene una rápida evolución, innovación y un alcance global como su locus, mientras que la economía tradicional de mercado fue constituida sobre un rígido modelo industrial con una orientación local, de la cual dependían empleos, mercados y todo el producto interno bruto de una nación. El surgimiento de la globalización quiere decir que el empleo, los mercados, la producción y las ganancias ahora son globales, desarticulando de ese modo generaciones de obreros que dependían de oportunidades económicas particulares.

La economía del conocimiento prefiere las estrategias basadas en la oportunidad, lo que garantiza una mayor participación en el mercado, antes que las estrategias basadas en los objetivos que son privilegiadas por la economía tradicional de mercado. La gran urgencia con la cual funciona la economía del conocimiento deja un mínimo de espacio para la reflexión profunda y para la creatividad como un novel derrotero; marginaliza la sabiduría de las mujeres e impide la innovación experimental espontánea o las oportunidades de experimentar fuera de ambientes controlados. El conocimiento indígena reconoce que la aplicación del intelecto y la creatividad están en función del beneficio comunitario y compartido sin que se limite el conocimiento de sus orígenes. Hoy en día existen áreas enteras de leyes, ya que el capital intelectual es considerado en términos monetarios y su valor inherente es medido en términos de la capacidad de generar ganancias. Este paradigma ha disminuido en gran medida la democratización del conocimiento. El acceso a la creatividad es promovido en tanto pueda generar ganancias antes que por su utilidad social, por el talento individual o por el ímpetu de crear y experimentar tan sólo por hacerlo para el bien común, como las mujeres lo han hecho por generaciones. 


\section{INTERPRETACIONES DE LA GLOBALIZACIÓN}

Los abordajes neoclásicos sugieren que la integración efectiva del conocimiento requiere el apalancamiento de cambios tecnológicos rápidos y la información excesiva, requiriendo grandes inversiones en tecnologías de la información. La fase inicial de colecta de información, procesamiento y evaluación, y la conversión resultante en conocimiento es una meta estratégica y central para el capital. Demoliendo barreras interdisciplinarias y facilitando una mayor disponibilidad y distribución de información, el conocimiento puede ser diseminado a través de la cadena de valor de la globalización. Por este medio, el conocimiento puede capitalizarse con mínima observación de la ética, de protección a los antiguos conocimientos o de una remuneración apropiada para la información y experticia de las mujeres. Subsecuentemente, la exploración de nuevas oportunidades de incorporar conocimiento está posibilitada por la creación de modalidades de descubrimiento de conocimiento meramente con fines de lucro.

Se puede discutir que la economía del conocimiento difiere de la economía del mercado tradicional en muchos aspectos clave:

- El asumir que la economía del conocimiento es abundancia antes que escasez. La información y el conocimiento pueden ser compartidos, utilizados y distribuidos sin agotarse a diferencia de la mayoría de los recursos tangibles, y de hecho pueden valorizarse más a través de su aplicación. Además no tienen competidores, ya que el consumo o información por un consumidor no prohíbe el consumo de lo mismo por otro. En este caso «puedes comer tu pastel y tenerlo».

- Los márgenes e impactos limitantes de localización se reducen en ciertas actividades económicas por la aplicación eficiente de «adecuadas tecnologías y métodos, mercados virtuales y organizaciones virtuales que ofrecen beneficios correspondientes, velocidad constante en operaciones relacionadas con el tiempo y el alcance global puede ser creado o, al contrario, reforzado en algunos otros aspectos económicos, por la creación de nichos de negocios alrededor de centros de conocimiento, tales como universidades y centros de 
investigación científica». Debe ser remarcado que los centros de conocimiento existieron mucho antes de la actual era de la economía del conocimiento.

- La interconectividad de las economías galvanizadas por la moderna comunicación facilita el rápido movimiento de nueva información y el acceso a las ventajas comparativas.

La información sigue a la demanda, y en esta era se procura regularla; legislarla o restringir su movimiento a las fronteras nacionales es no solamente inaprovechable para el capital, sino extremadamente problemático de imponer.

- La adición de valor no está restringida a los bienes tangibles y los servicios y productos y servicios mejorados por el conocimiento pueden incrementar exponencialmente el costo de esos productos.

- El valor y el precio de los productos y servicios están, entonces, relacionados con el contexto, tiempo y lugar; pueden variar en ciertos puntos. De hecho, la información puede tener diferente importancia para la misma persona en diferentes puntos, o inversamente, ser de una importancia extremadamente variante para diferentes personas en cualquier momento dado y su valor consignado depende marcadamente del contexto.

- El conocimiento tiene claramente el mayor valor intrínseco cuando está contenido en procesos o sistemas que cuando hay movilidad y facilidad de transferencia por medio del intercambio de ideas, conversaciones y estrategias como cuando la población intercambia ideas.

- En tanto que la globalización ve la reducción de escala como un mecanismo favorable y eficiente para reducir costos, muy pocas instituciones u organizaciones reportan sobre la eficacia o competencia cuando revisan su performance. Mientras que el capital humano es central para el conocimiento, raramente se ha referenciado en relación con el imperativo del lucro.

- En creciente medida, la globalización ve la comunicación como fundamental para el flujo de conocimiento. Ello necesita estructuras sociales y culturales centrales y otras relaciones sociales intrínsecas a las economías del conocimiento.

Recientemente, Ali Mazrui sugirió tres distintas maneras en que la globalización puede ser interpretada. La primera como una interdependencia

$100 \frac{\text { SEGUNDO SEMESTRE } 2015}{\text { MIGRACIÓN Y DESARROLLO NÚM. } 25}$ 
económica que atraviesa amplios espacios geográficos. La segunda se centra en la disponibilidad de información y su habilidad de viajar rápidamente a través de grandes espacios y distancias; finalmente la interpretación popular del mundo como una aldea global. Por otra parte, Mazrui sugiere que son identificables dos modelos de globalización, siendo éstos la globalización económica y la globalización cultural. Él presenta el análisis normativo de la globalización como la «interconectividad del mundo». Varios observadores y comentaristas señalan que la naturaleza de la globalización es "un desencuentro entre el poder político y el económico», y concuerdan en que los beneficiarios de tal diseño están en el Norte global. Este documento concuerda con la afirmación de que el colonialismo es una encarnación más temprana de la globalización.

Mazrui comparte el sentimiento y propone el argumento de que la palabra "globalización» es nueva, que la primera encarnación empezó hace siglos y fue conducida por cuatro grandes motores: religión, tecnología, economía e información para posibilitar el proyecto de construcción imperial incluyendo materia prima y expansión de mercados. La religión fue instrumentalizada para minar y desplazar las antiguas y diversas culturas de los colonizados. Esto fue garantizado por el proyecto vigente de no memoria y arregló el desplazamiento desde tierras antiguas para abrir camino para el saqueo físico, emocional, espiritual e intelectual de los colonizadores.

El proyecto de no memoria posibilitó la relativamente fácil remoción de pueblos antiguos de sus propios territorios históricos y geográficos, y con ello la remoción de la cultura e información, la explotación de sus herencias culturales en pro de nuevas tecnologías, mientras la concurrente denigración de los originarios los desplazó de sus territorios culturales para abrir caminos a favor de los colonos y su "desarrollo»; los objetos culturales y artefactos fueron saqueados y llevados lejos de los países colonizados.

\section{TRANSFORMACIÓN FUNDAMENTAL EN LA ECONOMÍA GLOBAL}

La economía de la información está basada en una transformación fundamental de la estructura subyacente de la economía política global. Varios 
académicos ahora sugieren que este cambio es tan fundamental que amerita el rótulo de cambio de paradigma tecnoeconómico. Este cambio refleja cambios en la ciencia, tecnología, organización de procesos de negocios, producción aprendizaje y entretenimiento. Aspectos de esta transformación incluyen: 1) la naturaleza de la compañía o empresa manufacturera; 2) la naturaleza cambiante de las dinámicas de negocios; 3) grandes cambios en las actividades empresariales de investigación y desarrollo; 4) fusión de tecnologías; y 5) inercia institucional. Estos cambios están afectando de cerca a todos los sectores del sistema global, incluyendo organizaciones intergubernamentales, el sector privado, organizaciones regionales, organizaciones no gubernamentales y Estados nacionales.

Esta era de cambios transformadora presenta un potencial de reestructuración de las relaciones de poder y el desarrollo de nuevas formas de desigualdad en el mundo. Es posible que la desigualdad en la economía de la información pueda ir más allá de una división entre los llamados países desarrollados y en vías de desarrollo para exacerbar las divisiones intranacionales. Específicamente, las divisiones podrían profundizarse entre los individuos que poseen el conocimiento, capacidades y habilidades para contribuir a la economía de la información global (sin importar su ubicación) y aquellos que no poseen tales capacidades.

\section{Negación del CONOCimiento indígena}

La globalización cultural aún está contribuyendo a la erosión de la cultura y lingüística de los pueblos. Muchos científicos, investigadores y comentaristas permanecen escépticos y rechazan el conocimiento indígena creyéndolo no documentado y no científico. Esto ha resultado en una predisposición negativa y desdeñosa contra las prácticas indígenas como los métodos tradicionales de medicina, educación, tecnología y agricultura. La razón proporcionada a menudo es que tales prácticas no tienen ninguna base científicamente probada. La información indígena es considerada inferior porque no está respaldada ni validada por métodos científicos.

$102 \frac{\text { SEGUNDO SEMESTRE } 2015}{\text { MIGRACIÓN Y DESARROLLO NÚM. } 25}$ 
Básicamente, la mayoría concuerda en que a pesar del progreso en la promoción de la centralidad del análisis de género en cualquier investigación social, todavía existe mucho por avanzar en la transformación social. Hay un sinnúmero de desafíos no resueltos en la estructuración de la contribución de las mujeres al conocimiento, y todavía ninguno de ellos se ha incluido en los desafíos del desarrollo y en una apropiada estructuración feminista. Elaborar un discurso sobre el desarrollo que sea una reiteración feminista amerita atención deliberada a una crítica del desarrollo de género y a los fundamentos sobre los cuales las aproximaciones al desarrollo son creadas. Esto incluye una recontextualización de la contribución de las mujeres y su impacto en los discursos sobre el desarrollo. Todo ello amerita una reflexión radical, investigación renovada y estudios de alternativas centradas en la gente.

Muchos científicos sociales e investigadores siguen preocupados tratando de comprender los sistemas de conocimiento indígena para utilizarlos en la resolución de los desafíos modernos u occidentales. Mucha gente joven de comunidades indígenas y pastoriles evitan sus propios sistemas tradicionales de conocimiento. Dada la creciente influencia de la cultura globalizada, esto es tan previsible como profundamente lamentable. Las jerarquías inherentes al conocimiento occidental son a menudo disonantes con el igualitarismo de algunos sistemas de conocimiento. El imaginario popular creado por la cultura occidental contribuye al desinterés que muchos jóvenes sienten hacia los sistemas de conocimiento indígena, pues éstos no son valorizados en los discursos de los medios masivos. En los últimos 40 años, la información y la sabiduría del conocimiento indígena de practicantes y comunidades se ha desplazado más allá desde el fetichismo de tiempos anteriores hacia la corriente principal de los discursos sobre la salud alternativa, agricultura y tecnología. Kenia ha contribuido notablemente con aproximaciones ingeniosas y originales en banca y tecnología ilustrando que la definición de conocimiento indígena requiere reformas dinámicas a medida que evoluciona.

Muchas comunidades y grupos de interés indigenista demandan crecientemente soberanía como una reacción contra la globalización. Una creciente crítica a los sistemas de conocimiento occidentales basados en la 
desigualdad está contribuyendo a una mayor atención hacia los múltiples senderos de conocimiento, información e innovación. En las jerarquías de la epistemología y ontología occidental, el conocimiento indígena ha sido devaluado. La búsqueda de ventajas lucrativas para alimentar el proyecto del capitalismo global y del conocimiento ha creado una demanda de innovación. Anteriormente, la literatura supuso que los pueblos indígenas requerían soluciones similares para salir del subdesarrollo; ello creó exclusiones para mucha gente porque las voces y luchas individuales fueron ignoradas.

La investigación, cultural y lingüísticamente, sobre diferentes estudiantes en las ciencias de la educación desafía las nociones tradicionales de WMS en cuanto a contenido, aprendizaje, enseñanza y evaluación (Lee y Greene, 1999). Ya no es aceptable por mucho tiempo tratar a diversos grupos como si fuesen homogéneos y compartiesen una experiencia única; en vez de ello, el estudio de lo indígena urge al mundo a tomar nota de su derecho y único lugar en la investigación multicultural. David Clark (2004: 203) señaló que los estudios de lo indígena son sobre «restablecer el bienestar a nuestras naciones».

\section{ÉTICA DE LA PROTECCIÓN DEL CONOCIMIENTO INDÍGENA}

Las complejidades de las formas de protección al conocimiento son a menudo intangibles o sustentadas comunitariamente y presentan algunos dilemas éticos de no protegerla. Ng'etich y Njoro (2005) resalta estos aspectos.

Los cambios en las formas de vida y de los modos de ser significan que las comunidades que no poseen documentación o procesos de conocimiento desaparecerán. Sus sistemas de conocimiento y filosofías podrían desaparecer con ellos a menos que sean documentados o registrados.

La escasez de experticia tecnológica y recursos financieros inhibe la capacidad de muchas comunidades de proteger su conocimiento y prevenir que sea explotado por las compañías orientadas al lucro. El problema es que muchas grandes compañías tienen la capacidad tecnológica para asistir la apropia-

$104 \frac{\text { SEGUNDO SEMESTRE } 2015}{\text { MIGRACIÓN Y DESARROLLO NÚM. } 25}$ 
ción y desarrollo de las innovaciones del conocimiento indígena. El riesgo es que ellas mismas vayan a apropiarse de la información o distorsionarla.

El riesgo del conocimiento indígena (CI) no documentado es que está disponible al dominio público y puede ser utilizado y aplicado por cualquier grupo que tenga los medios. La documentación de los sistemas de conocimiento indígena posibilitaría el desarrollo de una base de datos contra la cual las solicitudes de patentes deberían probarse.

Escritores como Ruiz (2004) han discutido los medios por los cuales las comunidades pueden asegurar y proteger el conocimiento indígena únicamente para su apropiación y así recibir sus enormes beneficios. El conocimiento puede ser un mecanismo dinámico de transición de las capacidades económicas y de desarrollo de las comunidades. Herramientas como las licencias de know-how ofrecen una protección más amplia para las comunidades mientras protegen sus beneficios autónomos de apropiación.

Varios autores, como Chisenga (2002), señalan que los países del Sur - especialmente las naciones africanas - contribuyen a internet con una cantidad poco importante de conocimiento o contenido. Internet está completamente dominada por Estados Unidos, Europa y Asia. Tales escritores han insistido en que estos países necesitan poner su propia información indígena en la red global para minimizar la convicción de la información occidentalizada para resolver y encaminar desafíos locales, y también tener acceso a innovaciones desarrolladas localmente. Además, la mayoría de los países africanos tienen un excedente de conocimiento indígena en contextos regionales, nacionales y aun locales. Esto puede ayudar a países, industrias y sociedades a desarrollar y generar riqueza, y esta información puede ser un medio para cerrar la brecha del conocimiento que parece existir.

A pesar de todo, surgen cuestiones muy importantes: żde quién es la responsabilidad de subir el contenido del conocimiento indígena en internet?, żcómo y qué comunidades tendrán acceso a su propio contenido?, y żlas comunidades con capacidades y autonomía tienen que controlar el acceso y el uso de tal contenido? 


\section{Migración y economía del conocimiento}

Un área de fundamental interés en el campo de la migración calificada es el discurso de la economía basada en el conocimiento (EBC) y la sociedad basada en el conocimiento (SBD) relacionado con el discurso acerca de la inmigración y la formulación de políticas en muchos países del Norte global. La acelerada capacidad del conocimiento y la información para cambiar radicalmente la economía y su impacto en la sociedad fue observada desde los años de 1950. Pero no asumió un papel central en la formulación de la política hasta los años de 1990. Casi todo el discurso está centrado en utilizar los recursos como inyecciones de capital para posibilitar el crecimiento económico. La jerarquía y discriminación de clase inherente a la globalización privilegian a la migración de mano de obra calificada que puede contribuir a la expansión económica. Esta perspectiva tiene muy poco interés por la disminución del conjunto de capacidades y conocimiento en los países de origen y por el impacto longitudinal en los países del Sur global. Ello hace parte del moderno componente del actual despojo de recursos de conocimiento, particularmente al Sur, por la cínica destreza del Norte.

La contextualización conceptual de ambos es necesaria para examinar su papel de apoyo a la economía global. Que la gente migra con conocimientos y sabiduría está normalmente reconocido en el contexto de la experticia profesional, pero la explotación del conocimiento de los pueblos africanos, sus habilidades y capacidades y su contribución a la economía global raramente se reconoce.

Países como Australia y Canadá, desde los noventa han orientado sus políticas de inmigración hacia los migrantes calificados. En Europa, Reino Unido ha llevado esta estrategia aún más lejos, privilegiando los sectores globalizados de finanzas y de las tecnologías de la información y la comunicación (TIC). Se ha planteado la idea de que el concepto dominante de la economía del conocimiento se ha acercado hacia una hiperglobalización, sugiriendo que el movimiento de cambio en la información puede alterar potencialmente el empleo, la producción y las formaciones sociales.

$106 \frac{\text { SEGUNDO SEMESTRE } 2015}{\text { MIGRACIÓN Y DESARROLLO NÚM. } 25}$ 
Un supuesto en la mayor parte del pensamiento predominante acerca de la economía del conocimiento es que el conocimiento gerencial, científico y tecnológico son las fuerzas que impulsan la globalización, la productividad y la creación de riqueza. El corolario es que lo que no se ajusta a este modelo de cambio tecnológicamente conducido no puede beneficiar la economía del Estado receptor, y por tanto debe evitarse la entrada a su territorio. $O$ si se le permite entrar, no puede disfrutar de los mismos derechos que aquellos que son útiles al crecimiento de la economía del conocimiento. De allí que la política de inmigración, especialmente en Europa, está crecientemente basada en derechos y senderos estratificados a la ciudadanía. Esta problemática propende a reproducir formas estratificadas de ciudadanía. Eventualmente crea una polarización entre los países que envían y su diáspora.

La visión de Castells es excesivamente especializada, y en ella propone unas cuantas entidades inconexas, la de las élites capacitadas, expertos y receptores de la economía del conocimiento que existen desde tiempos remotos y espacios indefinidos. Estas nuevas élites de la sociedad educada son los conductores de la nueva economía mundial, quienes hacen que continúe y la supervisan. Los discursos de globalización exhortan la libre movilidad de capacidades, conocimiento y trabajo, y la contracción del Estado-nación. Es un mundo en el cual la élite tiene la capacidad de mantener altos grados de movilidad y distribución irrestricta de su conocimiento, capacidades y beneficios. Tales «ciudadanos globales» en la clásica clasificación de migrantes (Calhoun, 2003) tienen la capacidad de consumir mercancías globales, a pesar de que un significativo número de ellos son pobres de tiempo y ricos de ingresos. Estas son las personas a las cuales las políticas migratorias van dirigidas para impulsar la economía del conocimiento, particularmente en el Norte global.

Esta perspectiva sugiere que la tendencia a posibilitar capacidades que puedan moverse sin esfuerzo y rápidamente a través de los sistemas globales, generalmente posibilitados por regulaciones estatales y ávidamente consumidos por otros en sociedades lejanas a los países de origen. Estas categorías de capacidades se mueven en gran medida sin restricciones por las regulaciones (v.g., las calificaciones no están rigurosamente confirmadas por 
ningún comité de expertos). Es más probable que los varones sean utilizados en capacidades profesionales mejor pagadas con muy pocas mujeres capaces de acceder a la cadena global de valor a través de la tecnología de la información o a «empresas altamente capacitadas». El gran apetito de la globalización por las capacidades para expandir el mercado y el capital ha influido discursos sobre la migración calificada. La clase emprendedora (tanto los utilizados en áreas privadas como los que han utilizado los negocios como un medio para relocalizarse) se volvió el centro de la literatura sobre migración calificada (Mahroum, 2001; Lavenex, 2002; OECD, 2002).

Los sectores de trabajo femenino (educación, salud, hostelería, trabajo social) crecieron poco en los años noventa. Como áreas de crecimiento, no incrementaron ni desarrollaron la economía. En el periodo desde los años cincuenta y sesenta los profesionistas médicos y trabajadores en salud se trasladaron en cantidades sustanciales al Norte global, que los percibió como una solución al costo y gastos de la seguridad social y deficiencias de los servicios de salud (Stasiulis y Bakan, 2005).

En el periodo de 1960 a 1972, la Organización Mundial de la Salud (OMS) halló que 5 por ciento de los profesionales de la salud estaban trabajando fuera de sus países de origen (Gómez-Mejía, Mccann y Page, 1979). Interesantemente, en los años noventa el paquete de ajuste conocido como neoliberalismo intentó reducir el gasto de seguridad social. Por ejemplo, Australia (Iredale, 2001), Canadá (Stasiulis y Bakan, 2005) y Reino Unido (Raghuram y Kofman, 2002) redujeron sus cuotas de estudiantes que eran especialistas, médicos internos y maestros. Coincidentemente, de manera creciente clasificaron estas ocupaciones como categorías escasas, las cuales calificaron practicantes para reasignaciones temporales y permanentes promoviendo una pequeña cantidad de migrantes en esos sectores. Las políticas de migración, como la canadiense, generalmente sustentaron los sectores predominantemente masculinos de la tecnología de la información (TI) y las finanzas. Como resultado de la búsqueda internacional de trabajo social y en salud, a finales de los noventa se vio un creciente y agudo recorte en la enseñanza, salubridad y trabajo social en el sur global, que siguió a la trayectoria global de la migración de Sur a Norte (Rosewarne, 2001). 
Además, estos patrones y trayectorias de capacidades están determinados por la cadena de valor global, incluyendo las funciones de terciarización de servicios, a pesar de no estar considerados como parte de las industrias masculinizadas, mejor pagadas, más bien próximas al amplio límite de las empresas sin fines de lucro o de área abierta. La minimización de esta clasificación en la literatura de la economía de la información, ideológicamente conectada con el sector de negocios (Hudson, 2006), se relaciona con una comparable subestimación de estos conjuntos en numerosas investigaciones sobre globalización.

Los activistas pro mujer en varios sectores (Benería y Sen, 1981; Mitchell y Bill, 2004; Petersen, 2003) han enfatizado la ausencia de consideración dada al trabajo en cuidados. Habitualmente, consignado como labores de casa, éstas subyacen a la llamada producción económica y lugar de trabajo, y además hacen un indicador útil de bienestar social. Debido a la ausencia de consideración del trabajo profesional, las pérdidas de fuentes de trabajo, en niveles senior en estos sectores ha promovido el desarrollo de trabajo migrante globalizado (Ehrenreich y Hochschild, 2003; Moya, 2007). Tanto trabajadores calificados (especialistas, ejecutivos, educadores y trabajadores sociales) como los semicalificados (cuidadores, trabajadores domésticos) son ocupaciones mayoritariamente feminizadas.

La globalización financiera es promovida por organizaciones transnacionales y multinacionales con la intención de ampliar su cuota de mercado y las vertientes de las materias primas e ideas creativas. En su mayoría, existe una tendencia a buscarlas en las naciones en vías de desarrollo. Luna sugiere que en realidad hay una increíble carrera a los bosques del mundo para aprender más sobre la capacidad terapéutica de las plantas. Mudiwa señala que los límites naturales son la columna vertebral de la supervivencia humana, los cuales están más presentes en las naciones en vías de desarrollo. Los límites naturales son establecidos normativamente para el beneficio de todo el mundo. La incongruencia surge cuando éstos son efectivamente procesados, los medicamentos por ejemplo tienen licencias, pero completamente inalcanzables para muchas comunidades de las mismas naciones donde la materia prima y las plantas se originan. 


\section{Globalización, conocimiento y RIESgo}

Adicionalmente a la amplitud de posibilidades que la tecnología ofrece, su uso es una empresa particularmente riesgosa cuando se la emplea en conjunto con el imperativo del lucro. Pese a que abre considerables posibilidades, el uso de la tecnología es, posiblemente con mucho, un gran impedimento para el desarrollo humano. Académicos como Ulrich Beck (1992: 13) señalan que:

La ganancia de poder a partir del "progreso tecno-económico» está siendo rápidamente opacada por la producción de riesgos. (Los riesgos en este sentido pueden ser vistos como la probabilidad de daño proveniente del cambio tecnológico y económico.) Los accidentes relacionados con la producción industrial, por ejemplo, pueden difundirse rápidamente más allá del contexto inmediato del que son generados. En otras palabras, los riesgos se tornan globalizados.

Además, dice:

[Los riesgos de la modernización] poseen una tendencia inherente hacia la globalización. Una universalización de accidentes acompaña a la producción industrial, independiente del lugar donde son producidos: las cadenas alimentarias conectan prácticamente a cada uno en la tierra entre sí. Se sumergen bajo las fronteras (Beck, 1992: 39).

Plantea preocupaciones adicionales diciendo:

[...] hay un efecto boomerang en la globalización de este tipo. Los riesgos les pueden aparecer a quienes los producen o se benefician de ellos. La observación básica subyacente tras todo esto es muy simple: todo lo que amenaza la vida en esta tierra también amenaza la propiedad e intereses comerciales de aquellos que viven de la mercantilización de la vida y sus requisitos. De esta manera surge una contradicción genuina, sistemática y creciente entre el lucro e intereses propietarios que aumentan el proceso de industrialización y sus consecuencias frecuentemente amenazadoras, las cuales ponen en peligro y expropian

$110 \frac{\text { SEGUNDO SEMESTRE } 2015}{\text { MIGRACIÓN Y DESARROLLO NÚM. } 25}$ 
posesiones y beneficios (sin mencionar la posesión y goce de la vida) (Beck, 1992: 39).

Esta es una de las paradojas centrales de lo que Beck ha denominado la "sociedad del riesgo». A medida que el conocimiento ha crecido, también el riesgo. De hecho, se podría señalar que las relaciones sociales, instituciones y dinámicas, dentro de las cuales se ha producido conocimiento han acentuado los riesgos relacionados. El riesgo ha sido globalizado.

\section{Conclusiones}

El conocimiento indígena y el conocimiento han sido profundamente afectados por la globalización. Una de las funciones más útiles de la globalización y la economía del conocimiento ha sido revelar la magnitud del valor que el CIA ha generado para el proyecto global. Además, la globalización ha negado el conocimiento indígena presentándolo como inválido y riesgoso salvo que fuera administrado por los —así denominados - expertos occidentales. La equivocada comercialización del conocimiento indígena ha alcanzado un punto de crisis, donde los emblemas considerados sagrados o aún tabúes por las sociedades son inapropiados y trivializados, como logotipos o vestimentas, y luego patentados sin permiso ni compensación. La globalización es esencialmente una nueva encarnación de la revolución industrial, determinada a alcanzar nuevas fronteras de lucro utilizando las ideas, capacidades y la movilidad de ambas. Además, la nueva revolución industrial está comprometida en crear los mayores beneficios para una minoría oligarca.

Es evidente que el Sur global debe crear medios más accesibles y efectivos en costo para proteger y extraer ideas y beneficios de esas ideas. Leyes sui generis, licencias de contratos y codificación del conocimiento indígena están entre los mecanismos de reforzamiento mutuo que potencialmente podrían asegurar las ideas africanas. Al contrario del paradigma anti-Estado inherente a la filosofía del mercado, esto requiere una amplia intervención del Estado y regulación gubernamental. Sudáfrica implementó una política SCI 
en 2004 para apoyar el crecimiento de la investigación y desarrollo. El gobierno nigeriano, en los últimos años, ha debatido la creación de un instituto de instrucción e investigación en medicina tradicional. Varios países surasiáticos han creado una biblioteca digital catalogando el conocimiento tradicional de la región y creando leyes contra el plagio. Es notable el caso de India, que ha desarrollado una biblioteca digital de conocimiento tradicional, usada como modelo por muchos otros países.

La ontología de existencia es la piedra angular del CIA y el recuerdo de la sabiduría es su esencia. A pesar del asalto corporativo al conocimiento indígena, la movilidad de la capacidad humana y las ideas del Sur o Norte, muchas iniciativas necesarias en el mundo en vías de desarrollo están surgiendo para proteger y promover el conocimiento indígena contra la globalización.

\section{Bibliografía}

Beck, Ulrich (2000), What Is Globalization? Cambridge, Polity Press, 2000. (2001), «Living Your Life In A Runaway World. Individualization, Globalisation And Politics», en Anthony Giddens y W. Hutton, The Edge. Living With Global Capitalism, 1a. ed., Londres, Vintage.

Benería, Lourdes (2008), «The Crisis of Care, International Migration, and Public Policy». Feminist Economics, 14, núm. 3, pp. 1-21.

Burwell, Catherine y Megan Boler (2008), "Calling on the Colbert Nation: Fandom, Politics and Parody in an Age of Media Convergence», Electronic Journal of Communication, 18, núm. 2.

Calmoun, Craig (2003), «"Belonging” in the Cosmopolitan Imaginary», Ethnicities, 3, núm. 4, pp. 531-553.

CAPRA, Fritjof (1989), Uncommon wisdom, Flamingo.

Castells, Manuel (2015), "Information Technology And Global Capitalism», en Anthony Giddens, The Edge. Living With Global Capitalism, 1a. ed., Londres, Vintage.

Chisenga, Justin (2002), «Indigenous Knowledge: Africa's Opportunity to Contribute to Global Information Content», South African Journal of Libraries and Information Science, 68, núm. 1, p. 16.

$112 \frac{\text { SEGUNDO SEMESTRE } 2015}{\text { MIGRACIÓN Y DESARROLLO NÚM. } 25}$ 
Cogburn, Derrick (1998), "Globalisation, Knowledge, Education and Training in The Global World», Global Information Infrastructure Commission, Chicago, Unesco, internet: 16 de julio 2015.

CORTADA, James W. (1998), Best Practices in Information Technology: how Corporations get the Most Value from Exploiting their Digital Investments, Prentice-Hall.

DAES, Erica-Irene (2015), «Some Observations And Current Developments on the Protection of the Intellectual Property of Indigenous Peoples», Wipo Roundtable On Intellectual Property And Indigenous Peoples (Mesa redonda sobre propiedad intellectual de los pueblos indígenas), Ginebra, N.p., 1998, internet: 16 de julio de 2015.

Dondolo, Luvuyo (2005), "Intangible Heritage: the Production of Indigenous Knowledge in Various Aspects of Social Life», Indilinga African Journal of Indigenous Knowledge Systems: A Cross-Pollination and Critique, 4, núm. 1, p. 110.

Ehrenreich, Barbara y Arlie Russell Hochschild (2003), Global Woman: Nannies, Maids, and Sex Workers in the New Economy, Macmillan.

Frank, Thomas (2000), One Market Under God, Nueva York, Doubleday, 2000.

Gee, James Paul, Glynda A. Hull y Colin Lankshear (1996), The New Work Order, Boulder, Colo, Westview Press.

Giroux, Henry A. (2001), Stealing Innocence, Nueva York, St. Martin's Press.

Gómez-MejíA, Luis R. Joseph E. Mccann, y Ronald C. Page (1985), «The Structure of Managerial Behaviors and Rewards", Industrial Relations: A Journal of Economy and Society, 24, núm. 1, pp. 147-154.

Iredale, Robyn (2001), "The Migration of Professionals: Theories and Typologies», International Migration, 39, núm. 5, pp. 7-26.

Kiggundu, John (2007), «Intellectual Property Law and the Protection of Indigenous Knowledge», en Indigenous Knowledge Systems and Intellectual Property in the Twenty-First Century: Perspectives from Southern Africa, pp. 26-47.

Klein, Naomi (2000), No Space, No Choice, No Jobs, No Logo: Taking Aim at the Brand Bullies, 1a. ed., Nueva York, Picador USA, p. 490.

LaveneX, Sandra (2002), «EU Enlargement and the Challenge of Policy Transfer: the Case of Refugee Policy», Journal of Ethnic and Migration Studies, 28, núm. 4, pp. 701-721.

LeE, Mo-Yee y Gilbert J. Greene (1999), «A Social Constructivist Framework for Integrating Cross-Cultural Issues in Teaching Clinical Social Work», Journal of Social Work Education, 35, núm. 1, pp. 21-37. 
Leys, Colin (2001), Market-Driven Politics: Neoliberal Democracy and the Public Interest, 1a. ed., Londres, Verso, (FALTA INFORMACIÓN)

Machlup, Fritz (1962), The Production and Distribution of Knowledge in the United States, vol. 278, Princeton University Press.

Mahroum, Sami (2001), "Europe and the Immigration of Highly Skilled Labour», International Migration, 39, núm. 5, pp. 27-43.

Maila, M. W. y C. P. Loubser (2003), «Emancipatory Indigenous Knowledge Systems: Implications for Environmental Education in South Africa», South African Journal of Education, 23, núm. 4, p. 276.

MaILA, Mago W. y Johannes Seroto (2013), «Indigenous Ways of Knowing and Sustainable Development in Higher Education", Journal of Human Ecology, 43, núm. 2, pp. 165-172.

MASOGA, Mogomme (2005), «South African Research in Indigenous Knowledge Systems and Challenges of Change», Indilinga African Journal of Indigenous Knowledge Systems: a Cross-Pollination and Critique, 4, núm. 1, p. 15.

MazruI, Ali (2001), Pan-Africanism and the Origins of Globalisation, Binghamton University, internet: 16 de julio de 2015.

Mitchell, William y Anthea Bill (2004), «Spatial Dependence in Regional Unemployment in Australia», p. 312.

MoAHI, Kgomotso H. (2006), "Documenting Indigenous Knowledge Systems In Africa: Prospects And Challenges», ESARBICA Journal: Journal of the Eastern and Southern Africa Regional Branch of the International Council on Archives, p. 241.

Mudiwa, Morries (2002), "Global Commons: The Case Of Indigenous Knowledge, Intellectual Property Rights And Biodiversity», en The Commons in An Age of Globalisation, Victoria Falls, N.p., Print.

NeL, Philip J. (2005), «Indigenous Knowledge Systems: Contestation, Rhetorics and Space», Indilinga African Journal of Indigenous Knowledge Systems: a Cross-Pollination and Critique, 4, núm. 1, p. 2.

NG'Etich, Kibet A. y Kenya Njoro (2005), «Indigenous Knowledge, Alternative Medicine and Intellectual Property Rights concerns Kenya», 11th General Assembly, Maputo, Mozambique, pp. 6-10.

Odora Hoppers, Catherine A. (2005), «Indigenous Knowledge and the Integration of Knowledge Systems: Towards a Conceptual and Methodological Framework», en Odora-Hoppers (ed.). Indigenous Knowledge and the Integration of

$114 \frac{\text { SEGUNDO SEMESTRE } 2015}{\text { MIGRACIÓN Y DESARROLLO NÚM. } 25}$ 
Knowledge Systems: Towards a Philosophy of Articulation, Claremont, Estados Unidos, New Africa Books.

Thomas, Pradip y F. Nyamnjoh (2007), «Intellectual Property Challenges in Africa: Indigenous Knowledge Systems and the Fate of Connected Worlds», en Indigenous Knowledge Systems and Intellectual Property in the Twenty-first Century: Perspectives from Southern Africa, p. 12.

Raghuram, Parvati y Eleonore Kofman (2002), «The State, Skilled Labour Markets, and Immigration: the Case of Doctors in England», Environment and Planning A34, núm. 11, pp. 2071-2090.

Romer, Paul M. (1986), «Increasing Returns and Long-Run Growth», Journal of Political Economy, 94 (5), pp. 1002-1037.

Ruiz, M. (2004), Policy Briefs: National and Regional Laws to Protect IK Related to Genetic Resources. SciDev. Accesible en http://www.scidev.net/en/health/traditional- medicine/policy-briefs/national-and-regional-laws-to- protect-ik-related-t. html internet: 3 de enero de 2007.

Sen, Amartya (2002), «How to Judge Globalism», The American Prospect, 13, núm. 1, pp. 1-14.

Stasiulis, Daiva (2004), «Hybrid Citizenship and what's Left», Citizenship Studies, 8, núm. 3, pp. 295-303.

Stasiulis, Daiva K. y Abigail B. Bakan (2005), Negotiating Citizenship: Migrant Women in Canada and the Global System, University of Toronto Press. 
